\title{
From Molecular Dating to Functional Materials
}

Christer Aakeröy, ${ }^{a}$

${ }^{a}$ Department of Chemistry, Kansas State University, Manhattan, KS, 66506, USA e-mail: aakeroy@ksu.edu

When molecules transition from solution into the condensed phase, their behavior and properties are to a large extent governed by intermolecular interactions. Despite the fact that such chemical bonds are relatively weak and reversible they are critically important to solubility, thermal and mechanical stability, optical properties, and many other key performance parameters of modern materials. Consequently, if we want to acquire the ability to design and construct new materials through a bottom-up approach that is both robust and versatile, we need a better understanding of the structural consequences, and balance between, intermolecular forces. In addition, we also need to establish more reliable and tangible connections between molecular structure and materials properties. In this presentation we will examine how several fundamental physical properties of a substance can be modified and 'dialed-in' through the use of cocrystallization technologies that are firmly anchored in a fundamental understanding of intermolecular forces [1-3].

\section{References}

[1] Gunawardana, C.A. \& Aakeröy, C.B., (2018). ChemComm, 54, 14047-14060.

[2] Sandhu, B. et al. (2018) ChemComm, 4657-4660.

[3] Aakeröy, C.B. et al. (2015) Chem. Eur. J., 21, 11029-11037 